http://jmscr.igmpublication.org/home/ ISSN (e)-2347-176x ISSN (p) 2455-0450

crossref DOI: https://dx.doi.org/10.18535/jmscr/v8i1.136

\title{
A Study on Maternal and Perinatal Outcome in Epilepsy Complicating Pregnancy
}

\author{
Authors \\ Prameeda $\mathbf{P R}^{1}$, Shahida $\mathbf{J}^{\mathbf{2}^{*}}$, Seetha $\mathbf{P M}^{3}$ \\ ${ }^{1}$ Assistant Professor in O\&G, SreeGokulam Medical College, Thiruvananthapuram \\ ${ }^{2}$ Associate Professor in O\&G, Government Medical College, Alappuzha \\ ${ }^{3}$ Retired Professor and HOD, Government Medical College, Kollam \\ *Corresponding Author
}

Shahida J

Associate Professor in O\&G, Government Medical College, Alappuzha, India

\begin{abstract}
Background: Incidence of seizure disorder in women attending antenatal clinics is estimated to be 0.3$0.5 \%$ of all births. These pregnancies are a challenge to patient and clinician alike, the double burden of seizures and the antiepileptic drugs [AED] exposure are responsible for the poorer outcome of infants born to mothers with epilepsy.
\end{abstract}

Materials and Methods: This was a descriptive study conducted in the antenatal outpatient clinic, antenatal wards and labour room of a tertiary care hospital over a period of one year. All pregnant women with pre-existing or recently diagnosed epilepsy after 20 completed weeks of gestation, a total of 126 patients were recruited. All patient details were collected using a structured questionnaire after getting their consent. Data were analysed using SPSS version 17.0 to obtain Chi square test and p value. The significance of the findings were interpreted.

Result: 126 pregnant WWE were studied over a period of one year. Among women on AED 51.7\% had seizures and $46 \%$ had statistically significant increase in frequency of seizures while women not on AED had $23 \%$ seizure and $20.6 \%$ had increased frequency of seizures during pregnancy. IUGR was statistically significant in AED group. Emergency CS $25.6 \%$ was statistically significant in AED group as compared to $10.3 \%$ among non- AED group, which could be due to higher induction rates among AED group.

Conclusion: Risk of maternal and fetal complications can be reduced with effective preconceptional planning, careful management during antenatal and post partum period.

Keywords: WWE women with epilepsy, AED anti epileptic drugs.

\section{Introduction}

Epilepsy is the second common chronic neurological disorder complicating pregnancy after migraine. About 2.5 million women in India suffer from epilepsy, with $52 \%$ of them being in the reproductive age group. Incidence of seizure disorder in women attending antenatal clinics is estimated to be $0.3-0.5 \%$ of all births. These pregnancies are a challenge to patient and clinician alike, the double burden of seizures and the antiepileptic drugs [AED] exposure are responsible for the poorer outcome of infants born to mothers with epilepsy ${ }^{1}$. Neurologists and obstetricians are increasingly faced with WWE during pregnancy. Majority of WWE who become pregnant have uncomplicated pregnancies and 
deliver healthy infants. Experimental and clinical studies have shown that seizures are influenced by female sex hormones, estrogen and progesterone. Hence in about $1 / 3$ rd seizures worsen and in the rest 2/3rd they either reduce or remain unchanged. So the management issues that need to be addressed in these pregnancies include preconceptional folic acid supplementation, the teratogenecity of anticonvulsant medication, obstetric complications, infant cure, breastfeeding, contraception and the long term outcome for $\mathrm{WWE}^{2}$.

The preconception management is the cornerstone in epilepsy care in WWE. A careful reappraisal of each case should ascertain the diagnosis, need for continued AED therapy, selection of appropriate AEDs, optimization of dosage and prescription of folic acid. During the pregnancy, the feta status need to be monitored with estimation of alphafetoproteins [AFP], and ultrasonography. Minor variations in anthropometric features have been observed in infants. Several institutions recommend oral vitamin $\mathrm{K}$ towards the end of pregnancy when enzyme inducing AEDs are prescribed because the later may potentially predispose the newborn to hemorrhagic disease, but recent reports indicate that such a risk is practically negligible. Besides WWE need to know that enzyme inducing AEDs may lead to failure of oral contraception. ${ }^{1}$

\section{Materials and Methods}

This was a descriptive study conducted in the antenatal outpatient clinic, antenatal wards and labour room of a tertiary care hospital over a period of one year. All pregnant women with preexisting or recently diagnosed epilepsy after 20 completed weeks of gestation, a total of 126 patients were recruited. All patient details were collected using a structured questionnaire after getting their consent. Demographic parameters like age, parity, socioeconomic status, education, occupation, etc. were noted. The onset and type of epilepsy was noted. Detailed neurological history was taken. Details about preconceptional counselling, periconceptional folic acid and regularity of antenatal checkups were assessed. Antenatal complications were looked for in every visit. Details of seizure characteristics and AED changes were studied. Routine blood and radiographic investigations were done. Antenatal corticosteroids were given routinely to all cases presenting with preterm labour. Onset of labour was noted-whether spontaneous, induced or an elective Caesarian section. Induction was needed in some cases for various obstetric indications.

Labour was watched for progress, fetal distress, MSAF, seizures, and PPH. Mode of delivery was noted. After delivery, a detailed neonatal examination was done- Apgar score, birth asphyxia, birth weight, gestational age, anomalies, and hemorrhagic diseases were excluded. NICU admissions were sought and noted when needed. All perinatal deaths were recorded. Patients were discharged on the 3rd day after vaginal delivery. Only those who had undergone caesarean section or whose babies required observation or NICU admission stayed back.

Data were analysed using SPSS version 17.0 to obtain Chi square test and $\mathrm{p}$ value. The significance of the findings was interpreted.

\section{Results}

\section{Demographic variables}

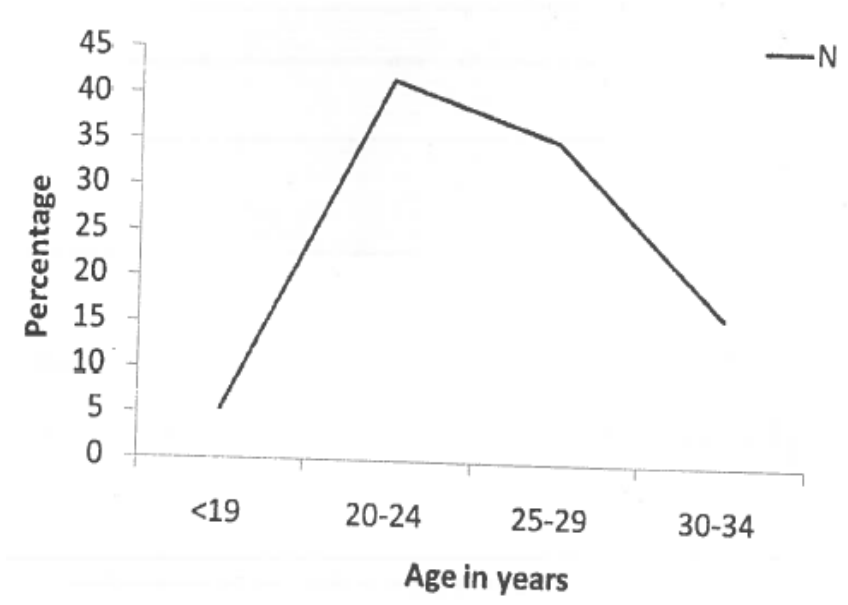

Figure 1: Distribution according to age $42.1 \%$ of patients belonged to 20-24 years age group followed by $35.7 \%$ to $25-29$ years age group. Mean age was 25.35 years and Standard deviation was 4.2. 
Table 1: Distribution according to educational background

\begin{tabular}{|l|c|c|}
\hline Education & N & Percentage \\
\hline Primary & 11 & 8.7 \\
\hline High School & 73 & 57.9 \\
\hline PDC & 20 & 15.9 \\
\hline Degree and above & 22 & 17.4 \\
\hline Total & 126 & 100.0 \\
\hline
\end{tabular}

Majority of my patients [57.9\%] had high school education.

Table 2: Distribution according to parity

\begin{tabular}{|l|c|c|}
\hline PARITY & N & Percentage \\
\hline Primi & 77 & 61.1 \\
\hline Para 1 & 42 & 33.3 \\
\hline Para 2 & 5 & 4.0 \\
\hline >Para 3 & 2 & 1.6 \\
\hline Total & 126 & 100.0 \\
\hline
\end{tabular}

Majority of women $61.1 \%$ belonged to primipara group followed by parity 1 .

Table 3: Distribution according to vitamin $\mathrm{K}$ prophylaxis

\begin{tabular}{|l|c|c|}
\hline Vit K taken & Frequency & Percent \\
\hline Taken & 95 & 75.4 \\
\hline Not taken & 31 & 24.6 \\
\hline Total & 126 & 100.0 \\
\hline
\end{tabular}

Vitamin $\mathrm{K}$ was taken in $75.4 \%$ women and all of them were on AED either antenatally or when reported in labour.

Table 4: Distribution according to maternal antenatal complications

\begin{tabular}{|c|c|c|c|c|c|c|c|c|}
\hline \multirow{2}{*}{$\begin{array}{l}\text { Antenatal } \\
\text { complications }\end{array}$} & \multicolumn{2}{|c|}{ AED } & \multicolumn{2}{|c|}{ No AED } & \multicolumn{2}{|c|}{ Total } & \multirow[t]{2}{*}{$\mathbf{X 2}$} & \multirow[t]{2}{*}{$\mathbf{P}$} \\
\hline & $\mathrm{N}$ & $\%$ & $\mathrm{~N}$ & $\%$ & $\mathbf{N}$ & $\%$ & & \\
\hline Hyperemesis & 7 & 8.0 & 3 & 7.6 & 10 & 7.9 & 0.00 & 0.90 \\
\hline Threatened abortions & 3 & 3.4 & 0 & 0 & 3 & 2.3 & 1.38 & 0.24 \\
\hline UTI & 9 & 10.3 & 4 & 10.2 & 13 & 10.3 & 0.00 & 0.98 \\
\hline Abruption & 1 & 1.1 & 0 & 0 & 1 & 0.7 & 0.45 & 0.50 \\
\hline IUGR & 13 & 14.9 & 1 & 2.5 & 14 & 11.1 & 4.17 & 0.04 \\
\hline GDM & 8 & 9.2 & 5 & 12.8 & 13 & 10.3 & 0.38 & 0.58 \\
\hline GHTN & 11 & 12.6 & 5 & 12.8 & 16 & 12.6 & 0.00 & 0.97 \\
\hline Placenta preavia & 0 & 0 & 1 & 2.5 & 1 & 0.7 & 2.25 & 0.13 \\
\hline Infections & 1 & 1.1 & 1 & 2.5 & 2 & 1.6 & 0.46 & 0.79 \\
\hline Breech & 2 & 2.3 & 1 & 2.5 & 3 & 2.3 & 0.01 & 0.12 \\
\hline Oligohydramnios & 8 & 9.2 & 6 & 15.4 & 14 & 11.1 & 1.04 & 0.31 \\
\hline Polyhydramnios & 3 & 3.4 & 1 & 2.5 & 4 & 3.1 & 0.07 & 0.79 \\
\hline
\end{tabular}

UGR was more in women in AED as compared to the no AED group which was statistically significant.

Table 5: Distribution according to onset of labour

\begin{tabular}{|l|c|c|c|c|c|c|c|c|}
\hline \multirow{2}{*}{ Onset of labour } & \multicolumn{2}{|c|}{ AED } & \multicolumn{2}{c|}{ No AED } & \multicolumn{2}{|c|}{ Total } & X2 & P \\
\cline { 2 - 9 } & $\mathrm{N}$ & $\mathbf{\%}$ & $\mathrm{N}$ & $\mathbf{\%}$ & $\mathbf{N}$ & $\%$ & & \\
\hline Spontaneous & 40 & 46.0 & 20 & 51.3 & 60 & 47.6 & 0.30 & 0.58 \\
\hline Induced & 38 & 43.7 & 9 & 23.1 & 47 & 37.3 & 4.89 & 0.02 \\
\hline Elective CS & 9 & 10.3 & 10 & 25.6 & 19 & 15.1 & 4.92 & 0.02 \\
\hline Total & 87 & 100 & 39 & 100 & 126 & 100 & & \\
\hline
\end{tabular}

Induction of labour was $43.7 \%$ in the AED group and elective CS was $25.6 \%$ in the no AED group which were statistically significant.

Table 6: Distribution according to mode of delivery

\begin{tabular}{|l|c|c|c|c|c|c|c|c|}
\hline \multirow{2}{*}{ Mode of delivery } & \multicolumn{2}{|c|}{ AED } & \multicolumn{2}{c|}{ No AED } & \multicolumn{2}{c|}{ Total } & \multirow{2}{*}{ X2 } \\
\cline { 2 - 8 } & $\mathrm{N}$ & $\mathbf{\%}$ & $\mathrm{N}$ & $\mathbf{\%}$ & $\mathbf{N}$ & $\%$ & & \\
\hline Vaginal & 49 & 56.3 & 22 & 56.4 & 71 & 56.3 & 0.00 & 0.99 \\
\hline Instrumental & 3 & 3.4 & 2 & 5.2 & 5 & 3.9 & 0.21 & 0.65 \\
\hline Elective CS & 9 & 10.4 & 10 & 25.6 & 19 & 15.0 & 4.92 & 0.02 \\
\hline Emergency CS & 26 & 29.9 & 5 & 12.8 & 31 & 24.7 & 4.23 & 0.04 \\
\hline Total & 87 & 100 & 39 & 100 & 126 & & & \\
\hline
\end{tabular}

Emergency CS was $29.9 \%$ in the AED group which was statistically significant. 
Table 7: Distribution according to neonatal variables

\begin{tabular}{|l|c|c|c|c|c|c|c|c|}
\hline \multirow{2}{*}{ Bwt in kg } & \multicolumn{2}{|c|}{ AED } & \multicolumn{2}{c|}{ No AED } & \multicolumn{2}{c|}{ Total } & \multirow{2}{*}{ X2 } & \multirow{2}{*}{ P } \\
\cline { 2 - 8 } & $\mathrm{N}$ & $\mathbf{\%}$ & $\mathrm{N}$ & $\mathbf{\%}$ & $\mathbf{N}$ & $\%$ & & \\
\hline Birth asphyxia & 6 & 6.8 & 4 & 10.3 & 10 & 7.9 & 0.42 & 0.51 \\
\hline Apgar score <7@5, & 8 & 9.2 & 0 & 0 & 8 & 6.3 & 3.74 & 0.05 \\
\hline NICU admission & 11 & 12.6 & 5 & 12.8 & 16 & 12.7 & 0.00 & 0.97 \\
\hline Neonatal infection & 2 & 2.3 & 3 & 7.6 & 5 & 4.0 & 2.06 & 0.15 \\
\hline Hemnorrhagic disease & 1 & 1.11 & 1 & 2.6 & 2 & 1.6 & 0.34 & 0.55 \\
\hline Hyperbilirubinemia & 11 & 12.6 & 6 & 15.4 & 17 & 13.5 & 0.17 & 0.67 \\
\hline Anomalies & 10 & 11.2 & 3 & 7.6 & 13 & 10.3 & 0.42 & 0.51 \\
\hline
\end{tabular}

Among the studied newborn variables only Apgar Score $<7$ at 5 were statistically significant in the WWE on AED.

\section{Discussion}

\section{Demographic variables}

$42.1 \%$ and $35.7 \%$ of patients in the study belonged to 20 to 24 and 25 to 29 year age group, with a mean age of 25.35 years. $93.7 \%$ of the women were housewives, who belonged to low socioeconomic status $62.7 \%$. $57.9 \%$ of women were educated up to high school $61.1 \%$ of my patients were primiparas.

\section{Epilepsy parameters}

In the present study $56.3 \%$ patients had h/o childhood onset of epilepsy. $80.1 \%$ of women had localized type of epilepsy of which complex partial seizures were more common followed by simple partial seizures. Primary generalized seizures were present in $19.9 \%$ which included 4 cases of JME. Women with JME only one was on VPA others were on other AED.

\section{Maternal variables}

In this study there were $8 \%$ patients who had preconceptional counseling. There is no evidence to inform the content, methods of delivery or effectiveness of preconception counseling to improve pregnancy outcomes for WWE and their offspring. In the present study, the WWE were divided into AED and no AED groups and further into those on monotherapy and those on polytherapy according to the intake during their periconceptional period. It was noted that there were $69 \%$ of WWE un AED [59.5\% were on monotherapy, $9.5 \%$ on polytherapy of the total women in study] and $31 \%$ were not on AED.
Periconceptional folic acid was only taken by $34.4 \%$ of women on AED and only $7.6 \%$ of not on AED which was significant. This is mainly because of lack of preconceptional care.

In my study antenatal seizures were present in $51.7 \%$ of women on AEDs and that noted in WWE not on AED was only $23 \%$ which was significant.

In this study, WWE irrespective of AED use, there was $38 \%$ increase, $49.4 \%$ no change and $12.6 \%$ decrease in seizure frequency. WWE on AED also showed a significant increase [46\%] in seizure frequency when compared to those not on AED [20.6\%]. 18.4\% had a decrease and $35.6 \%$ had no change in seizure frequency in women on AED. 79.4\% women not on AED had no change in seizure frequency. Women on monotherapy had a $48 \%$ increase, $16 \%$ had a decrease and $36 \%$ had no change in seizure frequency. Women on polytherapy had a $33.3 \%$ increase, $33.3 \%$ had decrease and $33.3 \%$ had no change in seizure frequency but was not statistically significant. Most of the seizures occurred due to drug default. In this study Vitamin $\mathrm{K}$ was taken in $75.4 \%$ women and all of them were on AED. These patients were administered the vitamin $\mathrm{K}$ either in the antenatal period in third trimester, when admitted or at the onset of labour. Current report states that there also was no evidence to support or refute taking vitamin $\mathrm{K}$ in WWE on maternal enzyme-inducing AED [Aleksey 2010].

Regarding maternal obstetric complications, in my study there was the following complications: IUGR [11.1\%], GHTN [12.6\%], GDM [10.3\%], 
hyperemesis [7.9\%], h/o threatened abortion [2.3\%], UTI [10.3\%], oligohydramnios [11.1\%], polyhydamnios [3.1\%], abruption [0.7\%], breech [2.3\%], placenta praevia [0.7\%] in WWE. Among them IUGR was statistically significant in the AED group, the rest were comparable in both groups.

In this study, $56.3 \%$ women had a vaginal route of delivery in both groups and $39.7 \%$ had a CS, in which the emergency CS rates were statistically significant in the WWE on AED, all were done for obstetric indications. This could be due to higher induction rates. $3.9 \%$ Instrumental deliveries were seen in WWE in this study which is similar to a study in Canada which reported that same [Richmond et al. 2004].

\section{New born variables}

In my study $88.1 \%$ of women had a term gestation and $11.9 \%$ were preterm. AED may cause preterm birth through drug induced folate deficiency [Steegers-Theunissen RP 1994], through depression of thyroid function [Kaneko S 1991] or through epoxide formation [Jones KL 1989]. In this study, preterm delivery were noted in $11.5 \%$ and $12.8 \%$ in the AED and no AED groups, none was statistically significant. One reason that preterm labour may be higher in this study is that a large majority of patients belong to the low socioeconomic group.

In this study $6.3 \%$ of babies had a poor Apgar score [ $<7 @ 5$ all of which belonged to the AED group and was statistically significant. This was mainly seen in preterm deliveries. In this study there was $10.3 \%$ anomalies in WWE, it was comparable in both the AED [11.2\%] and no AED [7.6\%] groups.

\section{Conclusion}

Careful planning and management of any pregnancy in WWE is essential to increase the likelihood of a healthy outcome for mother and infant.

Although women who have epilepsy and are taking AEDs do have increased risks for maternal and fetal complications, these risks can be reduced with effective preconceptional planning and careful management during pregnancy and the postpartum period.

Although in this study majority of the women had a favourable outcome, maternal obstetric complications and anomalies were seen. Preconceptional counseling was inadequate. IUGR was noted significantly more in WWE on AED. Induction rates were significantly more in WWE on AED so was the emergency CS rates. Among the newborn variables APGAR score $<7$ @ 5' was statistically significant in the AED group. Supplement of folic acid periconceptionally and during pregnancy and use of AEDs with less potential for teratogenicity may help reduce the incidence of malformations. Educational efforts should be targeted to improve the management. A team effort including obstetrician and neurologist can ensure a healthy and fruitful life.

\section{Summary}

- Total of 126 WWE were included during one year study.

- $56.3 \%$ had childhood onset of seizures followed by adolescence onset.

- Majority of had localization type of epilepsy of which complex partial seizures were more common followed by simple partial seizures. There were also 4 cases of JME.

- Preconceptional counseling had been taken only by $8 \%$.

- Regular health seeking behaviour was noted in $93.7 \%$.

- Periconceptional folic acid intake was seen only in $22.2 \%$ overall in WWE.

- Outcome were analysed in those on AED [69\%] and those not on AED (31\%),they were further divided in those on monotherapy (59.5\% of all WWE] and those on polytherapy [9.5\%] according to periconceptional AED intake.

- Antenatal seizures were present in $43.2 \%$ of WWE almost similar to some studies. $51.7 \%$ of women on AEDs and $23 \%$ in WWE not on 
AED had antenatal seizures, which was significant. Though there was no difference with respect to monotherapy and polytherapy.

- WWE on AED also showed a significant increase [46\%] in seizure frequency when compared to those not on AED [20.6\%]. There was no difference with respect to monotherapy and polytherapy.

- There were no cases of status epilepticus in the study.

- $66.6 \%$ of WWE did not have a change in AED/dose. In WWE not on AED 6.3\% had to be started on monotherapy and $0.7 \%$ had to be put on polytherapy. In the monotherapy group, $5.5 \%$ had to be put on polytherapy. All WWE on polytherapy continued the same. $19.8 \%$ of WWE had to increase the dose of AED.

- Among the antenatal complications IUGR was statistically significant in the AED group, the rest were comparable in the other groups.

- Vitamin K was given to $75.4 \%$ women and all of them were on AED. These patients were administered the vitamin $\mathrm{K}$ either in the antenatal period in third trimester, when admitted or at the onset of labour. .,-

- Overall induction rate was $37.3 \%$ in WWE. Induction rate was statistically significant in the AED group [43.7\%] as compared to the no AED group [23.1\%] the most common reasons were GHTN, IIJGR, and oligohydramnios.

- Elective CS were significantly higher [25.6\%] in no AED group when compared to the AED group [10.3\%] most of it was for previous CS with $1^{\circ} \mathrm{CPD}$.

- $56.3 \%$ women had a vaginal route of delivery in both groups and $39.7 \%$ had a CS, in which the emergency $\mathrm{CS}$ rates were statistically significant in the WWE on AED, all were done for obstetric indications, could be due to higher induction rates.

- $88.1 \%$ of WWE had a term gestation and $11.9 \%$ were preterm delivery. None were significant in AED/ no AED/ monotherapy/ polytherapy. One reason that preterm labour may be higher in this study is that a large majority of patients belong to the low socioeconomic group.

- $8.4 \%$ postpartum seizures were noted which were comparable in all groups. These were due to sleep deprivation and drug default.

- 1ntrapartum and postpartum maternal complications were comparable in mono/polytherapy groups.

- Birth weights were comparable in AED/AED and mono/ poly groups with a mean of $2.9 \mathrm{~kg}$.

- $\quad 6.3 \%$ of babies had a poor Apgar score [<7 5'] all of which belonged to the AED group and was statistically significant, but that in the monotherapy/polytherapy groups were comparable.

- NICU admission [12.5\%], birth asphyxia [7.9\%], neonatal infections [4\%] and haemorrhagic disease [1.6\%] were comparable in all groups.

- In this study there was $10.3 \%$ anomalies in WWE, it was comparable in both the AED and no AED groups, but statistically significant in the polytherapy group $33.3 \%$ when compared to $8 \%$ of monotherapy group.

- All the anomalies noted in the no AED group were ASD. CBZ was one of the drugs in all WWE of polytherapy group with anomalies. Phenobarbitone and CBZ were the drugs taken by WWE in the monotherapy group.

- Perinatal death was $4 \%$ of total births, $2.4 \%$ were NNDs all of which belonged to the monotherapy group, $1.6 \%$ IUD, all of which belonged to the polytherapy group [16.6\%] and was significant.

- NNDs were- congenital diaphragmatic hernia, anencephaly and irieningomyelocoele with CSF leak.

- Mean duration of hospital stay after delivery was 6.5 days, it was comparable in AED and no AED groups but significant in polytherapy 4 group [16.6\%] due to preterm and anomalous babies. 


\section{Bibliography}

1. Meador K, Reynold MW, Crean S et al. Pregnancy outcomes in women with epilepsy: a systematic review and metaanalysis of published pregnancy registries and cohorts. Epilepsy Res. 2008 Sep;81(1):1-13.

2. Byrne BM, Sayed S, Turner MJ. Is information given to pregnant women with epilepsy adequate? Epilepsia. 1997; [Suppl.3]:266.

3. Thomas SV. Management of epilepsy in pregnancy.JPGM.2006;52(1)57-64.

4. Richmond JR, Krishnamoorthy P, Andermann E, Benjamin A. Epilepsy and pregnancy, an obstetric perspective. Am J Obstet Gynecol. 2004 Feb; 190[2]:371-9.

5. Steegers- Theunissen RP, Renier WO. Bonn GF,et al. Factors influencing the risk of abnormal pregnancy outcome in epileptic women: a multicentric prospective study. Epilepsy Res 1994;18[3]: 261-9.

6. Kaneko S. Antiepileptic drug therapy and reproductive consequences: functional and morphological effects. Reprod-Toxicol 1991;5[3]:179-98.

7. Jones KL, Lacro RV, Johnson KA, Adams J. Pattern of malformations in children of women treated with carbamazepine in pregnancy. N Engl. J Med.1989 Jun22;320 [25]:1661-66. 